der ersten Bebrïtungshälfte die Verhältnisse nicht sicher zu beurteilen sind, folgt vom 11. Tage ab das Natrium in seinen Veränderungen dem Kalium. Es zeigt wie dieses am 14. Tage mit $225 \mathrm{mg}-\%$ ein Minimum und steigt bis zum Ende des Untersuchungszeitraumes wieder an. Diese Ergebnisse stehen im Gegensatz zu anderen Untersuchungen, die für die erste Hälfte der Bebrütungszeit eine Zunahme und vom 12. Tage an eine rasche Abnahme des Natriums in der Allantoisflüssigkeit ergaben ${ }^{2}$ ). Ganz andersartig verhält sich das Calcium. Von $7 \mathrm{mg}-\%$ am 9. Bebrütungstag ausgehend verdoppelt es sich bis zum 14. Tag, dem Tag der Kalinm- und Natriumminima, und fällt dann bis auf $11 \mathrm{mg}-\%$ am 16. Tag ab.

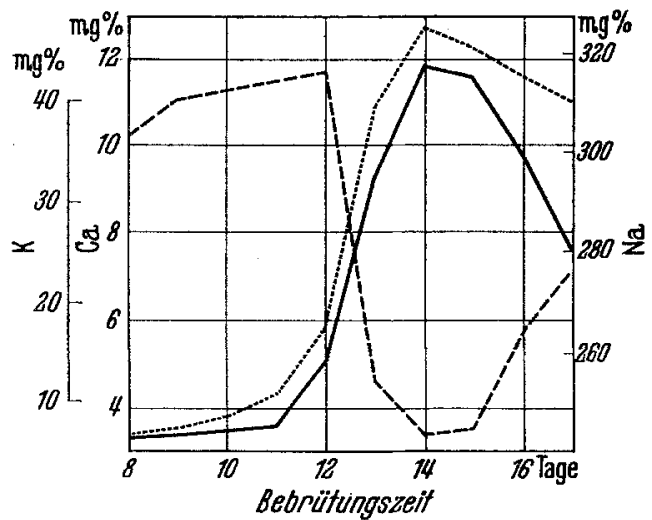

Fig. 1. Kalium- $(-\longrightarrow)$, Natrium-(- - $)$ und Calcium $(\cdots \cdots)$ gehalt in der Amnionflüssigkeit. Abszisse: 8., 9. Bebrütungstag usw. Ordinate: Kalium, Natrium- und Calciumgehalt in $\mathrm{mg}-\%$

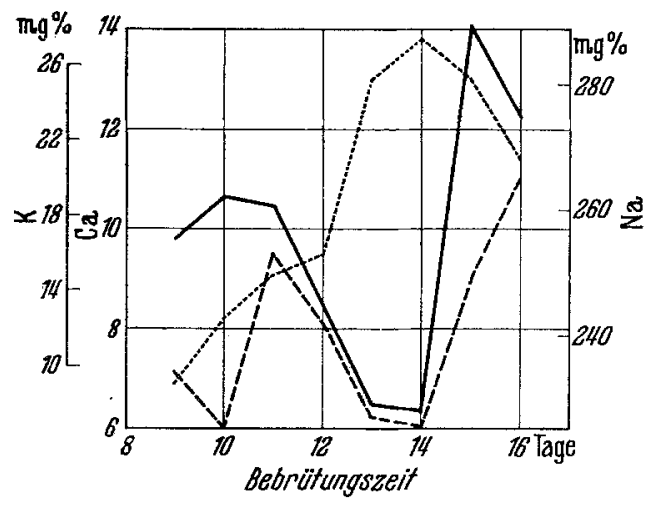

Fig. 2. Kalium-( $\longrightarrow$ ), Natrium $(--\longrightarrow)$ und Calcium $(\cdots \cdots \cdot)$ gehalt in der Allantoisflïssigkeit. Abszisse: 8., 9. Bebritungstag usw. Ordinate: Kalium-, Natrium- und Calciumgehalt in mg.\%

Außer dem bereits nachgewiesenen Einstrom von Proteinen und Aminosäuren in die Amnionflüssigkeit des Bruteies, der gleichfalls zwischen dem 11. und 13. Bruttag erfolgt und für die nächsten Tage Eiweißkonzentrationen von 11 bis $18 \mathrm{mg}$-\% zeigt'), ergibt sich, daß die Amnion- und auch die Allantoisflüssigkeit hinsichtlich des Elektrolytgehaltes zu Beginn der zweiten Bebrütungshälfte starke Veränderungen erfahren. In der Amnionflüssigkeit steigen Kalium und Calcium an, während das Natrium abnimmt. In der Allantoisflïssigkeit nehmen Kalium und Natrium ab; hier steigt der Calciumgehalt a.n. Alle Veränderungen beginnen zwischen dem 11. und 12. Bebrütungstag, erreichen am 14. Tag ihr größtes Ausmaß und lassen im letzten Drittel der Bebrütungszeit eine rückläufige Tendenz erkennen.

Neben dem steigenden Gehalt an Eiweiß und Aminosäuren als Ausgangsstoffe für die Plasmasynthese resultieren aus dem. Wechsel der Kalium-, Natrium- und Calciumkonzentrationen an den einzelnen Bruttagen unterschiedliche osmotische Verhältnisse, die für den Zeitpunkt der Beimpfung und das Wachstum von Bakterien und Viren im Brutei von Bedeutung sind.

Hygiene-Institut der Universität, Kiel (Divektov: Prof. Dr. med. H. GÄRTNER)

Eingegangen am 16. April 1960

G. WITt und D. SchyMa

1) WiTr, G., H. KNothe u. G. HannemanN: Naturwissenschaften 46, 266 (1959). - ${ }^{2}$ ) WALker, P. A.: Diss. Harward 1936. Zit. bei D. Thon, Inaug.-Diss. Kiel 1955.

\section{Zur Wirkung von Serumalbumin auf Lebermitochondrien}

Untersuchungen an frischen Rattenlebermitochondrien (Inzuchtstamm BD III), die wir nach Myers und SLATER ${ }^{1}$ ) präparierten, zeigten, daß der SerumalbumineinfluB komplex ist. Neben Fettsäuren ${ }^{2-5}$ ) sind noch andere Faktoren beteiligt.

Im System ohne Phosphatakzeptor wird die Geschwindigkeit der spontanen Succinatoxydation durch Dinatriumäthylendiamintetraessigsäure (EDTA) herabgesetzt. EDTA wird durch $\mathrm{Ca}^{++}$-Bindung wirksam ${ }^{6}$ ) $0,4 \mathrm{~g}-\%$ Serumalbumin ernied rigt in der gleichen Anordnung die Oxydationsgeschwindigkeit sogar noch etwas stär ker als EDTA. Setzt man EDTA und Serumalbumin gemeinsam $\mathrm{zu}$, so vergrößert sich die hemmende Wirkung nicht. Eine dem Serumalbumin äquivalente $\mathrm{Ca}^{++}-$Menge (konduktometrische Titration) hebt den hemmenden EinfluB des Serumalbumins auf. Die halbe Konzentration an $\mathrm{Ca}^{++}$vermindert den Serumalbumineffekt um etwa die Hälfte (Fig. 1). Nach unseren Befunden ist Serumalbumin als $\mathrm{Ca}^{++}$-Binder, wie es auch von EDTA angenommen ist, wirksam. Der $\mathrm{Ca}^{++}$ Effekt kann aber auch durch die Blockierung der Fettsäurebindung durch das Serumalbumin erklärt wer

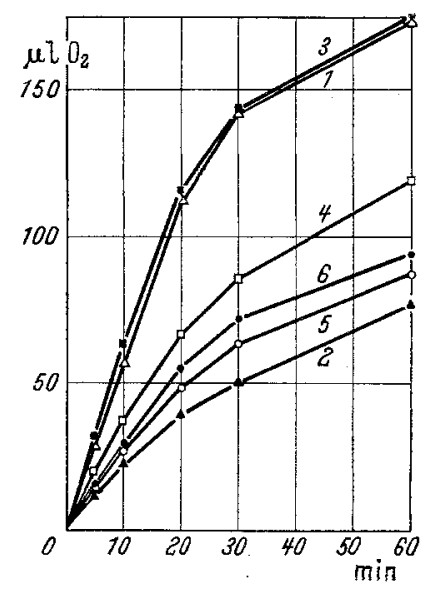

Fig. 1. Warburg-Technik, $t=37^{\circ}$ Gasphase $\mathrm{O}_{2}$. 0,5 ml Mitochondriensuspension in $0,25 \mathrm{~m}$ Saccharose $(N=0,085 \mathrm{~g}-\%)$ in einem Gesamtvolumen von $1,6 \mathrm{ml}$ : Kaliumphosphatpuffer $\mathrm{p}_{\mathrm{HI}} 7,4=1,7 \cdot 10^{-2} \mathrm{~m}$; DinatriumSuccinat $1,12 \cdot 10^{-2} \mathrm{~m} \cdot 1$ Ohme -itere Zusätze; 2 mit 0,4 or $0,4 \mathrm{~g}-\%$ Serumalbumin im Puffer; 3 mit $0,4 \mathrm{~g}-\%$ Serumalbumin $+3 \cdot 6$. $10^{-4} \mathrm{~m} \mathrm{CaCl}$ im Puffer; 4 mit $0,4 \mathrm{~g}-\%$ Serumalbumin $+1,8$. $10^{-4} \mathrm{~m} \mathrm{CaCl}$ im Puffer; 5 mit $28 \cdot 10^{-4} \mathrm{~m}$ EDTA im $2,8 \cdot 10-4$ EDTA in Puffer $6,4 \mathrm{git}$
$0, \%$ Serumalbumin im Puffer den, denn wir konnten nachweisen, daß die $\mathrm{Ca}^{++}$- Seife der Ölsäure durch Serumalbumin nicht mehr gebunden wird. Vermutlich sind also nicht die freien ungesättigten Fettsäuren, sondern ihre $\mathrm{Ca}^{++}-$Seifen die tatsächlich schädigenden Noxen.

Physiologisch-Chemisches Institut der Karl-Marx-Universität, Leipzig

Eingegangen am 13. Mai 1960

WOLFGANG KUNZ

1) Myers, D. K., u. E.C. Slater: Biochemic. J. 67, $558(1957)-$ 2) Lehninger, A. L., u. Le Mar F. RemmerT: J. Biol. Chemistry 234, 2459 (1959). - 8) Wojtczak, L., u. A.B. WoJTczak: Biochim. Biophys. Acta 39, 277 (1960). - 4) Ave-Dor, Y.: Biochim. Biophys. Acta 39, 53 (1960). - - 5) Hülsmane, W.C., W. B. Elltot u. E.C. Slater: Biochim. Biophys. Acta 39, 267 (1960). - b) Kunz, W., W. Friedel, F. Müller, Pham van Sô u. E. Strack: HoppeSeylers Z. physiol. Chem. 310, 265 (1958).

Der Auxin-Gehalt von Tradescantia-Blättern in Abhängigkeit von der Wellenlänge des Lichtes

In einer Arbeit meiner Schülerin I.M. HAHN ${ }^{1}$ ) über den Wirkstoffgehalt von Pflanzen, die bei ausschließlich künstlichem Licht herangezogen wurden, ist unter anderem der Auxin-Gehalt dieser in verschiedenfarbigem Licht kultivierten Pflanzen vergleichsweise qualitativ und quantitativ bestimmt worden. Jene Versuche ließen darauf schließen, daß die Aktivierung einiger Auxine in Blau- $(\sim 470 \mathrm{~nm})$, anderer Auxine in langwelligem Rotlicht $(>730 \mathrm{~nm}$ ) erfolgt, die Synthese der Auxine bzw, ihrer ,precursors" dagegen durch kurzwelliges Rot (660 nm) gefördert wird. Eine genaue Abhängigkeit der Aixin-Synthese vom $\lambda$ des Lichtes war aber aus diesen und anderen Untersuchungen noch nicht abzuleiten. Zur Klärung dieser Frage wurden jetzt folgende Versuche durchgeführt:

Unter die in Fig. 1 bezeichneten Schott-Filter (Lichtquelle: Leuchtstofflampen-Aggregat mit je 1 HN J de Luxe + $1 \mathrm{HNT}+1$ HNP Osram) wurden je 10 für die gesamte Versuchsserie ausgesucht gleichmäßige Tradescantia-Stecklinge für $7 d$ in Bechergläser mit Leitungswasser gestellt. (Tägliche Belichtungsdauer $16 \mathrm{Std}$ bei einer für alle Filter gleichmäßigen Lichtintensität von $5740 \mathrm{erg} / \mathrm{cm}^{2} / \mathrm{sec}$.) 